\title{
Spurious High Platelet Count by Automated Hematology Analyzer in Patient with Microcytic RBCS
}

\author{
Dr Rateesh Sareen ${ }^{1}$, Dr Menka Kapil ${ }^{2}$ \\ (Corresponding author) Consultant Pathology Santokba Durlabhji Hospital \& Research Center, Jaipur \\ Consultant Pathology Santokba Durlabhji Hospital \& Research Center, Jaipur
}

\begin{abstract}
We report a case of spurious elevation of platelet count on three consecutive occasions in a pediatric patient on six part Sysmex Hematology auto analyzer. Falsely low platelet counts are frequently reported in literature but falsely elevated Platelet counts are uncommon. The case emphasizes the important role of careful peripheral blood smear examination in case of high platelet counts. The blind folded reliance on 5 part analyzers in hematology should be discouraged so as to provide best patient care.
\end{abstract}

Keywords: Spurious platelet count, 5 part analyzer

\section{Introduction}

'Computers are magnificent tools for the realization of our dreams, but no machine can replace the human spark of spirit, compassion, love and understanding.'

The use of automated analyzer in hematology has become a rule rather than an exception. With the lack of legislation many laboratories rely heavily on automated analyzer and reports given to patients without any pathologist intervention or with a formal dummy signature. There is no doubt that these instruments have improved the accuracy of results with optimal quality control measures in laboratory. There is variety of conditions where automated parameters are fictitious. Such situation demands careful attention, because vital clinical management decisions may be taken using these erroneous results. It is therefore of paramount importance that both the laboratory personnel and clinician are aware of possibility of spurious data from automated instrument.

\section{Material method}

We present a case of artefactual rise in automated platelet count not only on single but on three consecutive occasions by Sysmex XN 1000 automated analyzer in an infant.

A case of 7 month old infant admitted with complaints of loose stools and vomiting since 6 days. The complete blood count reported by the Sysmex XN 1000 the six part differential automated analyzer used in our laboratory, revealed a hemoglobin of $6.8 \mathrm{~g} / \mathrm{dl}$; a total leukocyte count of $15.9 \times 10^{9} /$ liter, with a normal differential leukocyte count; mean corpuscular volume of $48.3 \mathrm{fl}$; mean corpuscular hemoglobin of $14.5 \mathrm{pg}$, and a mean corpuscular hemoglobin concentration of $30.6 \mathrm{~g} / \mathrm{dl}$. The platelet count was $2412 \times$ $10^{9} /$ liter. The abnormal high platelet count alarmed the pathologist and peripheral blood smear showed a platelet count of approximately $180-200 \times 10^{9} /$ liter, thereby resulting in discrepancy of nearly $2200 \times 10^{9} /$ liter compared with the automated value. Similar fictitious high platelet values were obtained for the next three days with machine value from $2790 \times 10^{9} /$ liter, $2332 \times 10^{9} /$ liter, $2129 \times$ $10^{9} /$ liter. The platelet counts by manual method were $225 \times$ $10^{9} /$ liter, $200 \times 10^{9} /$ litre, $180 \times 10^{9} /$ liter respectively. Such major variations on automated cell counters were not observed in any of the previous results.

\section{Discussion}

Spurious elevation of platelet count may be seen in patients with extensive burns, extreme microcytosis (as seen in $\mathrm{HbH}$ disease, microangiopathic hemolytic anemia, and red cell fragmentation in burns), micro-organisms like bacteria, fungi or yeast, hyperlipidemia, fragments of white blood cell (WBC) cytoplasm in patients with acute leukemia, hairy cell leukemia, and lymphomas or the presence of cryoglobulins ${ }^{1}$. This has the risk of under evaluation or treatment of a true thrombocytopenia as the patient's reported count could be spuriously normal or high. In our case the work up for spurious platelet count was done. There was no evidence of hemolysis of RBC as reticulocyte count $\&$ peripheral blood smear did not reveal any Fragmented Red cells. The blood culture and urine culture were negative thus ruling out infection as a cause of spurious platelet count as they are one of the reasons for falsely high platelet count. Cryoglobulinemia was ruled out by incubation of blood. Microcytosis was the only factor that could have resulted in inaccurate platelet counting in our case as it was severe with MCV 48-50 ft and with absence of other causes for falsely elevated platelet count. Therefore it is an eye opener for users where the general population has high incidence of iron deficiency anemia or thalassemia because both conditions produce microcytosis . Thus relying only on automated analyzer will produce misleading platelet counts $^{2}$. The impedance method for platelet count estimation at low MCV values will not provide accurate platelet count, instead a false high platelet count shall potentially affect platelet transfusion decisions for patients. This fact is established by study by Ninama et al., who reported that the impedance method was not always reliable for assessing platelet counts in cases of severe microcytosis after

\section{Volume 6 Issue 1, January 2017




\section{International Journal of Science and Research (IJSR) \\ ISSN (Online): 2319-7064}

Index Copernicus Value (2015): 78.96 | Impact Factor (2015): 6.391

comparing platelet counts obtained by the impedance method on the CELL-DYN ${ }^{\mathrm{TM}} 3700$ (Abbott Laboratories) analyser with those obtained from a manual technique with ammonium oxalate. ${ }^{3}$ Similarly, Pan et al. showed that the impedance method overestimated platelet counts in microcytic samples using the XE $2100^{\mathrm{TM}}$ automated analyser (Sysmex Corp.). ${ }^{4}$ The degree of microcytosis and fictitious increase in platelet count is not well established. This makes it extremely important for pathologist while reporting high platelet count solely on the basis of automated analyzers particularly in cases of microcytosis.

The automated hematology analyzers use two basic methods - optical density and electrical impedance to count platelets ${ }^{5}$. The optical platelet count is obtained by a two-dimensional analysis that estimates the complexity and density of platelets represented as a cytogram of the light intensity at $7^{\circ}$ and $90^{\circ}$ angles. Whereas impedance platelet count uses hydrodynamic focusing and single-dimensional histogram analysis to count the platelets based on their size. But one must be aware of limitations of both methods. Microcytic or fragmented red blood cells, cell debris, white cell fragments and giant platelets are situations where these automated analyzers give erroneous results due to their in ability to differentiate between platelet \& non platelet particle. Immunological methods using flow cytometry technology have been developed that use conjugated monoclonal antibodies directed against specific platelet antigens such as cluster of differentiation CD 41 and CD6. There are numerous studies in literature where platelet counts have been falsely reported as low counts by automatic analyzer and the counts increased on subsequent manual platelet estimation. But our observation of falsely high platelets by automated analyzer is less frequently reported in literature. At the same time such variation in platelet count on automated analyzers expose the automation technology and re enforce the importance of peripheral blood smear examination while reporting a complete blood count.

\section{Conclusion}

The trend in modern day pathology is to lessen the need to review blood smears, both by high output laboratories and by small laboratories with scarcity trained manpower. It is necessary that the sample with unexpected blood count in relation to the clinical settings or those that generate specific flags necessity a careful review of blood smear. The Standard operating procedure of automated analyzers must include definite guidelines on situations in which blood smear review is mandatory. This will help in reducing spurious results.

\section{Acknowledgements}

Dr G N Gupta Head of Department of Pathology \& Dr Rohit Jain Consultant Pathology, Santokba Durlabhji Memorial Hospital \& Research Center, Jaipur for their support and cooperation.

\section{References}

[1] Kakkar N, John M J, Mathews A, Chawala R. Spurious platelet count due to cryoglobulins in a patient with smoldering myeloma. Indian J Pathol Microbiol 2014; 57:476-9.

[2] Alkindi S, Al Zadjali S, Al Madhani A, Daar S, Al Haddabi H, Al Abri Q, et al. Forecasting hemoglobinopathy burden through neonatal screening in Omani neonates.Hemoglobin. 2010; 34:135-44. doi: 10.3109/03630261003677213.

[3] Ninama NJ, Shah NK. Impedance platelet count in severe microcytosis: Study of 161 patients. NHL J Med Sci. 2014; 3:32-6.

[4] Pan LL, Chen CM, Huang WT, Sun CK. Enhanced accuracy of optical platelet counts in microcytic anemia. Lab Med. 2014; 45:32-6. doi: 10.1309/LM7QPULDM5IHBO3L.

[5] Briggs C, Harrison P, Machin SJ. Continuing developments with the automated platelet count. Int J Lab Hematol. 2007; 29:77-91. doi: 10.1111/j.1751553X.2007.00909. 